Cipango $\begin{aligned} & \text { Cipango } \\ & \text { Cahiers d'études japonaises }\end{aligned}$

19 | 2012

Le Japon et le fait colonial II

\title{
La révolution chez Fukuzawa et la notion de jitsugaku
}

Fukuzawa Yukichi sous le regard de Maruyama Masao

Isabelle Lefebvre

\section{CpenEdition}

Journals

Édition électronique

URL : https://journals.openedition.org/cipango/1718

DOI : $10.4000 /$ cipango. 1718

ISSN : 2260-7706

Éditeur

INALCO

Édition imprimée

Date de publication : 30 octobre 2012

Pagination : 79-91

ISBN : 978-2-85837-204-7

ISSN : 1164-5857

\section{Référence électronique}

Isabelle Lefebvre, "La révolution chez Fukuzawa et la notion de jitsugaku », Cipango [En ligne], 19

2012, mis en ligne le 24 avril 2014, consulté le 30 juin 2021. URL : http://journals.openedition.org/ cipango/1718; DOI : https://doi.org/10.4000/cipango.1718

Ce document a été généré automatiquement le 30 juin 2021.

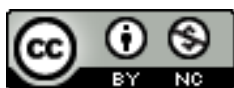

Cipango est mis à disposition selon les termes de la Licence Creative Commons Attribution - Pas d'Utilisation Commerciale 4.0 International. 


\section{La révolution chez Fukuzawa et la notion de jitsugaku}

Fukuzawa Yukichi sous le regard de Maruyama Masao

Isabelle Lefebvre

1 Au regard de la connaissance commune au Japon de l'illustre figure de l'époque Meiji qu'est Fukuzawa Yukichi, on peut se demander quel intérêt l'historien des idées qu'a été Maruyama Masao a trouvé à reprendre la notion de jitsugaku (実学, «études pratiques »1 $)^{1}$ par laquelle Fukuzawa Yukichi se fit connaître avant d'être célébré comme le «Voltaire du Japon». En 1947, l'année où le philosophe Maruyama Masao écrivit l'article traduit ci-dessous, il ne semblait a priori plus utile d'analyser ce que devait être le contenu des études selon celui qui fut «le rénovateur de l'éducation à l'époque Meiji ». En effet, le changement d'orientation proposé par Fukuzawa du contenu à donner aux études à travers la critique et le rejet des études confucéennes sans lien avec la pratique était reconnu comme une révolution accomplie vers l'utilitarisme et le réalisme. C'est cependant vers un retournement de cette vision commune sur l'apport de Fukuzawa à l'histoire des idées au Japon que nous conduit Maruyama. Pour comprendre la spécificité de l'intérêt que ce dernier lui a porté, nous retracerons quelques traits de l'œuvre des deux auteurs : Fukuzawa, l'un des premiers Japonais à aller dans le monde occidental y chercher l'essence de sa culture à l'époque Meiji, et Maruyama, historien des idées, penseur des principales étapes de la modernisation de son pays et témoin des grands évènements du $\mathrm{xx}^{\mathrm{e}}$ siècle.

\section{Fukuzawa et la rupture avec la tradition confucéenne}

2 Fukuzawa (1835-1901) a consacré sa vie et son œuvre tant à la recherche des fondements de la civilisation occidentale que des moyens de les transmettre par l'éducation. Au moment de la Restauration de Meiji, en 1868, alors âgé de trente-trois ans, il avait déjà ouvert sa propre école, baptisée cette année-là du nom sous lequel elle est devenue l'illustre université Keiō Gijuku. Il devait publier l'année suivante L'État de 
l'Occident ${ }^{2}$ pour se consacrer ensuite à ses principales réflexions sur l'éducation, dont est issu entre autres son célèbre Gakumon no susume (L'Appel à l'étude), publié en $1872^{3}$.

Le récit de sa vie nous permet de comprendre l'ancrage de sa détermination à trouver les principes de la civilisation occidentale. Exclu de l'enseignement primaire réservé aux enfants de samouraï, il a été sensibilisé tôt à la question des inégalités sociales ${ }^{4}$. Parvenu malgré ces obstacles au titre de professeur de néerlandais des vassaux du domaine de Nakatsu, il obtint les moyens de séjourner à plusieurs reprises aux ÉtatsUnis et en Europe. Son apport à l'histoire de la pensée au Japon reste pour tous celui d'un nouveau prototype d'étude fondé sur l'affirmation de l'égalité fondamentale de tous les êtres humains et sur la conscience que seule l'éducation permet le développement de l'autonomie et d'une Nation prospère, elle-même indépendante ${ }^{5}$. Ce point de départ se trouve formulé dès l'ouverture de son Appel à l'étude 6 .

4 C'est aussi par sa participation à l'ensemble du "mouvement vers l'Aufklärung »" et à la réflexion sur les droits du peuple sous-jacente à ce mouvement que Fukuzawa est associé à la rupture avec l'enseignement traditionnel confucéen. Dans Tsūzoku kokkenron $^{8}$ (Discours sur les droits du peuple ; 1878), paru quatre ans avant la traduction du Contrat social par Nakae Chōmin 中江兆民 (1847-1901), le chef de file du « Mouvement pour la liberté et les droits civiques » (Jiyū minken undō 自由民権運動), Fukuzawa, à la différence de ce mouvement, ne reconnaît pas de caractère naturel aux droits du peuple. Si chez lui la conscience collective doit toujours primer sur la conscience individuelle, c'est pour développer l'accord entre l'indépendance nationale et celle $\mathrm{du}$ peuple contre l'assujettissement à l'ordre naturel traditionnel, et non par conservatisme envers la tradition confucéenne visant à maintenir l'harmonie de la société selon cet ordre.

\section{La continuité d'une tradition dans « la révolution vers les études pratiques »}

5 Pour Maruyama, cette rupture avec la tradition confucéenne que marquent incontestablement l'œuvre et l'appel aux études pratiques de Fukuzawa ne vient cependant pas contredire son inscription dans ce que l'on peut appeler une «tradition de l'appel au retour à la pratique». Se détourner des pures spéculations théoriques pour aller vers les études en relation avec la vie réelle est une attitude récurrente depuis avant même la nouvelle tradition confucéenne des Song (960-1279) en Chine, désignée aussi par le terme de Shushigaku 朱子学, école de Zhu Xi (1130-1200). On sait que le renouveau du confucianisme en Chine suit une période de mise en lumière de la spécificité de chacune des trois voies, confucianisme, taoïsme et bouddhisme, après l'assimilation du bouddhisme dans la pensée aux $\mathrm{v}^{\mathrm{e}}$ et $\mathrm{VI}^{\mathrm{e}}$ siècles'. Les trois voies seront comprises plus tard, notamment sous la dynastie des Ming (1368-1644) par Wang Yangming 王陽明 (1472-1529) comme conduisant par des chemins différents au même objectif, l'accomplissement des vertus, qui ne sont plus l'apanage des Saints de l'antiquité, mais qui sont potentiellement identiques en tout homme. Pour Wang Yangming, la question n'est plus d'ordre cognitif, mais pratique : il ne s'agit plus de savoir quelle est notre nature et d'acquérir une connaissance livresque, mais, la potentialité en tout homme étant posée comme équivalente, de savoir comment devenir un saint. On sait également que le renouveau du confucianisme ainsi opéré a commencé à se développer au Japon avec Fujiwara Seika 藤原惺窩 (1561-1619). 
Maruyama qualifie la nouvelle tradition confucéenne des Song qui se développe sous les Tokugawa (1600-1868) tout d'abord à travers l'école Shushigaku, de "rationalisme moraliste fondé sur la continuité entre l'homme et le ciel $»^{10}$. Cette continuité se trouve exprimée dans la formule de Fujiwara Seika :

La Voie du Ciel, c'est le Li (principe). Quand ce Li est dans le Ciel, il est appelé «Voie du Ciel ». Quand il est présent dans l'esprit humain, mais qu'il n'est pas encore exprimé dans aucune action, il est appelé « nature » de l'homme ${ }^{11}$.

6 Maruyama s'est attaché à retracer les étapes du développement au Japon de ce renouveau confucéen ainsi que les principaux éléments déterminant son éclatement dans l'article faisant l'objet de cette présentation, ainsi que dans son Essai sur l'histoire des idées politiques au Japon. Parmi ces éléments, notons ceux que reprend son article, à savoir la séparation progressive entre théorie abstraite et pratique ainsi que la progressive reconnaissance d'une discontinuité entre norme et nature.

7 Dans son Essai, il repère un premier moment sur le chemin qui a conduit à la sortie du confucianisme dans le passage aux Kogaku (古学: 《études anciennes ») avec Yamaga Sokō 山鹿素行 (1622-1635), Itō Jinsai 伊藤仁斎 (1627-1705) et Kaibara Ekiken 柏原益軒 (1627-1705). En effet, ce nouveau type d'études marque une première étape dans la primauté accordée aux faits et en l'occurrence à l'examen des textes anciens plutôt qu'aux théories spéculatives. Avec Ekiken, fondateur de la botanique au Japon, si l'étude du principe reste primordiale, c'est dans le but d'arriver aux choses et aux faits mêmes.

L'œuvre d'Ogyū Sorai (1766-1728), par son apport d'une méthodologie, conduit à une remise en question plus marquée du rationalisme confucéen. Sa critique porte déjà les caractéristiques de celle de Moto.ori Norinaga (1730-1744) fondateur du mouvement nativiste (Kokugaku 国学) pour lequel la Voie deviendra volonté divine, marquant ainsi le passage à un positivisme objectif puisque la Voie ne consistera et ne se révèlera plus que dans les marques des faits qu'il faut étudier.

9 En instaurant, dans la théorie comme dans la pratique, une séparation entre la politique et les autres disciplines, Ogyū Sorai a brisé la continuité de principe qui était au fond de la pensée du confucianisme. En posant la nécessité d'étudier la langue et son évolution pour comprendre la Voie par les textes anciens, il a montré que l'essentiel de l'étude doit en rester aux faits et non aux argumentations et spéculations, et mis ainsi parallèlement en lumière le caractère inconnaissable de la Voie.

\section{Le caractère révolutionnaire de l'appel aux études de Fukuzawa}

10 Maruyama montre que, par rapport aux appels au retour à la pratique qui l'ont précédé, la caractéristique de l'appel de Fukuzawa ne consiste pas tant dans le fait d'être destiné au peuple que dans l'importance que son auteur accorde à la physique issue du cartésianisme par opposition à la connaissance du monde de la nature issue des études confucéennes. Comprise comme point central du développement du savoir occidental, et par conséquent de ce que doivent être selon lui les études, la «physiquemathématique » l'intéresse surtout pour l'indépendance de l'esprit vis-à-vis de la nature qu'elle présuppose.

11 En effet, Fukuzawa, cité par Maruyama, considère que la supériorité de l'Occident tient en deux points incontournables pour le développement de l'humanité et dont l'Orient a manqué, à savoir, "pour le monde physique, les mathématiques, et pour le monde de 
l'esprit, l'autonomie ». Fukuzawa n'a pas tant posé comme centre et objectif de la connaissance la physique que lancé un appel à l'attitude à son origine. Là réside selon Maruyama le caractère révolutionnaire de l'appel de Fukuzawa aux études pratiques (jitsugaku). Or on peut caractériser cette attitude à l'origine de la physique par l'effacement qu'elle présuppose des prétentions de l'esprit à une connaissance hors de l'examen des faits, autrement dit, à une connaissance comme soumission face à la nature.

\section{L'approfondissement du sens de la pratique}

S'il s'agit bien, dans l'appel à l'étude à partir de l'attitude à l'origine de la physique, d'un appel à une pratique, ce n'est pas simplement à celle de la vérification par l'expérience d'hypothèses, mais aussi plus fondamentalement à celle d'un acte de la volonté s'appliquant à laisser de côté les spéculations pour s'adapter à l'ordre extérieur, objectif, des faits. Ainsi, si l'on peut parler de "pratique » pour caractériser la démarche expérimentale, c'est en premier lieu parce que par la soumission aux faits eux-mêmes rejoint la pratique du non-mental. C'est en second lieu la capacité qu'elle génère d'agir sur la réalité qui caractérise la physique comme pratique et en ce sens, pour Maruyama, il est juste de considérer la démarche de Fukuzawa comme fondamentalement pragmatique.

Cet appel à l'esprit à l'origine de la physique que Maruyama voit au cœur de l'appel de Fukuzawa se révèle donc bien paradoxalement un appel à la pratique dans le plein sens de ce terme par opposition à ce que l'on peut considérer comme pures constructions de l'esprit. Autrement dit, ce qui se révèle dans le besoin de retourner à des études directement liées à la vie elle-même, c'est la nécessité de sortir du monde jusqu'au bout subjectif qu'est le monde mental.

Avant d'aborder cette sortie du monde subjectif dans laquelle réside pour Maruyama l'intérêt et la profondeur de la signification de la révolution de Fukuzawa, revenons sur la pluralité de sens que prend le terme jitsu et que nous avons traduit par " pratique » dans l'expression jitsugaku (littéralement, études vraies, réelles).

Les études jitsugaku auxquelles appelle Fukuzawa sont, comme nous l'avons vu, des études pratiques tout d'abord dans le sens où l'esprit $\mathrm{y}$ acquiert son autonomie paradoxalement par le fait même de se soumettre à l'ordre objectif de la nature, ensuite dans celui où il est appel à l'expérience et à l'expérimentation, et encore dans le sens où cette attitude renvoie au vide des spéculations ou constructions purement mentales, et enfin dans le sens où cette étude est repérée comme celle même qui génère l'efficacité ou « capacité à agir sur le réel ».

\section{L'évolution des idées au Japon et la naissance de la subjectivité}

16 L'intérêt que Maruyama a porté à l'œuvre de Fukuzawa ne provient pas seulement d'un souci d'historien de ne pas laisser de côté ce moment de rupture avec les fondements de la pensée traditionnelle, mais aussi de sa recherche des clés tant du changement que de la continuité dans l'histoire des idées au Japon. Selon la formule d'Uno Shigeki ${ }^{12}$, Maruyama repère une «opposition incessante entre les prototypes et les facteurs 
innovants de la pensée japonaise ». En s'inscrivant simultanément dans une paradoxale continuité avec l'un des prototypes de la tradition et une innovation radicale, l'appel à la pratique offre une illustration de cette incessante opposition. Dans la section suivante, nous nous efforçons, en suivant Maruyama, de comprendre l'évolution dans laquelle cet appel s'inscrit et de saisir en son cours la naissance de la subjectivité.

\section{De l'éthique de la Voie à la différenciation entre subjectivité et objectivité}

17 L'intérêt de Maruyama pour la révolution chez Fukuzawa de la notion de jitsugaku relève aussi de sa recherche des fondements du monde des valeurs. Engagé par l'armée en 1945, il fut profondément marqué par la défaite du Japon et dans un article publié l'année suivante qui le rendit célèbre, «Logique et psychologie de l'ultranationalisme $»^{13}$, pour caractériser l'organisation et la structure du régime impérial, il utilise l'expression "régime de l'irresponsabilité ». Cette formule nous révèle la conscience qu'il avait de la nécessité d'une interrogation sur le lien entre l'origine des fascismes et l'insuffisance de fondement des valeurs. Dans l'article traduit ici sur la révolution chez Fukuzawa de la notion de jitsugaku, il a dégagé pour la réflexion sur le fondement de l'éthique une différence essentielle entre les études $d u$ renouveau confucéen et celles issues de l'esprit de la physique.

\section{L'ordre social, fondement de l'éthique et de la nature}

Les études du renouveau confucéen sont basées sur un principe reliant l'homme, le ciel et la terre et désigné comme Voie. Puisque la Voie unifie les relations entre les hommes et la compréhension de la nature, la nature ne peut y être pensée comme extérieure au sujet. Par conséquent, d'un côté, la nature est fondamentalement spirituelle et de l'autre, l'esprit n'est pas considéré dans la position de sujet, mais dans celle d'élément de la nature. Cette attitude est tautologique en ce sens que l'on suppose dans l'ordre de la nature des valeurs sociales en affirmant par exemple la supériorité du ciel sur la terre, et que l'on voit au fondement de l'ordre social, l'ordre de la nature. Par ailleurs, puisque l'ordre social et celui de la nature sont perçus comme des ordres naturels et immuables, le bien suprême ne peut résider ailleurs que dans l'union avec l'environnement.

19 Si le caractère tautologique de cette attitude n'est pas perçu comme tel, c'est nécessairement parce qu'un fond commun à ces deux ordres rend possible leur renforcement mutuel. Ce fond commun ne peut être autre que le principe de l'unité de la «Voie du Ciel » et de « la Voie de l'homme » qui vient servir de support aux relations sociales hiérarchiques comme à l'étude du monde de la nature. Toute étude produite au sein de cette unité est nécessairement une étude de la Voie qui ne peut elle-même porter que sur la recherche de l'éthique. À partir de ce rapport de l'homme au monde, ni la subjectivité, ni la physique comprise comme une étude de la nature en tant que telle, n'ont donc pu se constituer puisque la spécificité du rapport de l'homme au monde n'est pas objectivé en l'être humain, mais dans un ordre social dépourvu de norme extérieure à lui-même.

C'est en prenant conscience de sa subjectivité dans l'opposition à son environnement naturel que l'esprit, pour la première fois, se distancie des lois et des règles de la 
société. On peut dire avec Maruyama que s'il n'avait pas lui-même conscience que la critique d'un point de départ dans les principes hiérarchiques faisait naître l'opposition entre la subjectivité et le monde de la nature, Fukuzawa avait vu que dans le monde oriental cette opposition était impossible sans une rébellion contre l'ordre des couches sociales.

\section{Renforcement de la subjectivité et objectivation de la nature et de la société}

21 Cette analyse d'une étape clé de la naissance de la subjectivité au Japon donne un nouvel éclairage à l'histoire européenne du processus d'opposition de l'esprit en tant que sujet et de la nature en tant qu'objet. On peut repérer avec Maruyama différentes étapes dans ce processus au sein de l'histoire occidentale : tout d'abord l'opposition aristotélicienne entre la forme et la matière, puis les études s'appuyant sur la logique scolastique dérivée elle-même des dogmes de l'Église comme de la logique aristotélicienne. Dans la logique scolastique, on trouve, de même que dans les études confucéennes, une hiérarchisation de la nature en miroir avec celle de valeurs spirituelles. En venant retirer à la nature toute potentialité "spirituelle", la logique cartésienne s'est parachevée dans la compréhension d'une nature purement mécanique au sein de laquelle les valeurs ont été éliminées. C'est en se soumettant à une nature objective que l'esprit a renforcé son caractère actif et s'est accaparé la position du sujet.

Cette analyse de Maruyama révèle l'importance que Fukuzawa, en faisant de la physique un modèle idéal d'étude et en établissant celle-ci comme noyau central de l'attitude de l'esprit, accordait non seulement à l'approfondissement du sens de la pratique, mais aussi au renforcement du caractère actif de l'esprit.

\section{L'enjeu d'une pratique centrée sur le renforcement du sujet par sa soumission à l'ordre objectif}

En s'opposant à son environnement pour se soumettre à un ordre objectif, l'esprit cultive la nature objective et, en la «technicisant", il reforme objectivement son environnement. Le passage d'un ancien à un nouveau type d'études pratiques apparaît dès lors comme celui de l'adaptation à la réalité et à sa transformation. Peut-on conclure pour autant que c'est à raison que l'on a surtout vu en Fukuzawa un pragmatique? Maruyama souligne son caractère pragmatique dans le fait que c'est dans la démarche scientifique que Fukuzawa a trouvé le lien profond qu'il recherchait entre les études et la vie pratique quotidienne, à savoir qu'en ne fuyant plus dans aucun compromis facile avec la réalité, l'esprit peut surmonter les difficultés de la vie quotidienne présente tout en formant l'avenir. Mais il montre aussi que ce n'est pas tant pour la vie dans un environnement donné que Fukuzawa recherche l'utilité, que pour le dépassement des limites de cet environnement.

Maruyama a montré à travers Fukuzawa que c'est en transformant l'objet selon l'expérience que le sujet se pose activement en tant que tel. Il a ainsi fait apparaître l'anticipation mutuelle de la nature physique et de l'autonomie du sujet. Il a également montré que ce processus aboutissait à l'objectivation du sujet dans l'enchaînement mécanique des causes et des effets. Selon lui, si Fukuzawa a eu pleinement conscience qu'ainsi, au lieu de libérer la vie humaine conformément à l'espoir du mouvement vers 
l'Aufklärung, la science pourrait finir par nuire au développement libre de la vie en tant que telle, son appel aux études pratiques témoigne de la force de sa foi en une harmonisation possible entre la science et la vie. L'analyse de Maruyama éclaire ainsi pleinement la convergence entre l'idéal du mouvement vers l'Aufklärung et l'appel aux études pratiques.

\section{BIBLIOGRAPHIE}

\section{Édition du texte de la source}

MARUYAMA Masao, Fukuzawa ni okeru jitsugaku no tenkai. Fukuzawa Yukichi no tetsugaku kenkyū josetsu 福沢に於ける実学の展開、福沢諭吉の哲学研究序説, in Maruyama Masao senshū 丸山真 雄選集, vol. XVI, Tōkyō, Iwanami shoten, 1997, 2004, p. 108-131.

\section{Traductions}

KumAZAWA Banzan, Questions sur la Grande Étude (Daigaku wakumon), trad. et comment. JeanFrançois Soum, Tōkyō, Maison Franco-japonaise, 1995, 233 p.

\section{De Fukuzawa}

«L'Appel à l'étude [Gakumon no susume 学問のす > め] - livre premier », trad. et prés. Christian Galan, in Yves-Marie Allioux (dir.), Cent ans de pensée au Japon, vol. 1, Philippe Picquier, 1996, p. 7-26.

«L'Appel à l'étude [Gakumon no susume 学問のす > め] - livre deuxième », trad. et prés. Christian Galan, Daruma - Revue internationale d'études japonaises, n² 2, automne 1997, p. 259-266.

« Description des écoles de Kyōto [Kyōto gakkō no ki 京都学校の記] », trad. Christian Galan, in collectif, Traduction et étude critique de textes de réflexion de l'ère Meiji, Paris, INALCO, 2006, non paginé.

La vie du vieux Fukuzawa racontée par lui-même [Fukuōjiden 福翁自伝], trad. et annot. Marie-France Tellier, Paris, Albin Michel, 2007, 398 p.

Plaidoyer pour la modernité. Introduction aux œuvres complètes [Zenshūjoron 全集序論], trad. et intro. Marion Saucier, CNRS Éditions, 2008, 143 p.

\section{De Maruyama}

« Les intellectuels dans le Japon moderne [Kindai Nihon no chishikijin 近代日本の知識人]», trad. Jacques Joly, in Cent ans de pensée au Japon, tome II, Philippe Picquier, 1996, p. 273-332.

《La Pensée de l'État Meiji [Meiji kokka no shisō 明治国家の思想]», trad. Morvan Perroncel, Ebisu, nº 32, Tōkyō, Printemps-été 2004, p. 85-121.

Essai sur l'histoire des idées politiques au Japon [Nihon seiji shisō shi kenkyū 日本政治思想史研究], trad. Jacques Joly, P.U.F., 1996, 229 p.

Studies in the Intellectual History of Tokugawa Japan [Nihon seiji shisōshi kenkyū 日本政治思想史研究], trad. Mikiso Hane, University of Chicago Press, 1975, 383 p. 


\section{Études}

BLACKER Carmen, The Japanese Enlightement: a Study of the Writings of Fukuzawa Yukichi, Cambridge University Press, 1964, 252 p.

CHENG Anne, Histoire de la pensée chinoise, Éditions du Seuil, 2002, 650 p.

GIRARD Frédéric, HORIUCHI Annick, MACÉ Mieko (dir.), Repenser l'ordre, repenser l'héritage. Paysage intellectuel du Japon (XVII ${ }^{e}$-XVIII ${ }^{e}$ siècles), Droz, Genève, 2002, 524 p.

HAMON Claude et TsCHUDIN Jean-Jacques (dir.), La Nation en marche. Études sur le Japon Impérial de Meiji, Arles, Philippe Picquier, 1999, 206 p.

KERSTEN Rikki, Maruyama Masao and the Search for Autonomy, Routledge, London, 1996, 289 p.

SAUCIER Marion, « D’une école de rangaku à une université moderne - La naissance de Keiō gijuku », in Annick Horiuchi (dir.), Éducation au Japon et en Chine - Éléments d'histoire, Éditions les Indes savantes, Paris, 2006, p. 99-118.

Uno Shigeki, « La Modernisation politique au Japon et l'idée de subjectivité chez Maruyama Masao », Ebisu, n³2, Printemps-été 2004, p. 67-83.

NisHiKaWA Shunsaku, «Fukuzawa Yukichi », Perspectives : revue trimestrielle d'éducation comparée, Unesco, Bureau international d'éducation, vol. XXIII, n 3-4, sept.-déc. 1993, p. 501-515.

Soum Jean-François, Nakae Tōju (1619-1691) et Kumazawa Banzan (1619-1691). Deux penseurs de l'époque d'Edo, Paris, Collège de France, Institut des Hautes Études Japonaises, 2000, 505 p.

STEVENS Bernard, « Un regard japonais sur la modernité. La pensée politique de Maruyama », Esprit, Février 2005, p. 117-133.

\section{NOTES}

1. D’une manière générale, le terme jitsugaku 実学 désigne les études qui concernent le quotidien et la pratique, en opposition aux études dans lesquelles dominent la conceptualité spéculative et abstraite. Du point de vue historique, c'est en réaction à la systématicité abstraite et métaphysique de la pensée de Zhu Xi introduite au Japon, qu'est apparu, sous les Tokugawa, avec la nécessité de relier les études à la pratique, le terme jitsugaku. La volonté de renforcement du système du shogunat a abouti à n'utiliser ce terme que par rapport aux études techniques. Il est convenu de dire qu'avec Fukuzawa, le terme jitsugaku, que nous traduisons par «études pratiques ", oppose l'utilité quotidienne et ordinaire des hommes aux études vides ou à l'étude de la poésie classique. Tetsugaku jiten 哲学事典, Heibonsha 平凡社, 1971. C'est à l'encontre de cette opposition qui reste superficielle que s'inscrit l'article de Maruyama.

2. 西洋事情, 10 vol., 1866-1870.

3. «L'Appel à l'étude [Gakumon no susume 学問のす 〉 Galan, in Yves-Marie Allioux (dir.), Cent ans de pensée au Japon, vol. 1, Philippe Picquier, 1996, p. 7-26. «L'Appel à l'étude [Gakumon no susume 学問のす 〉 め - livre deuxième », trad. et prés. Christian Galan, Daruma - Revue internationale d'études japonaises, n 2, automne 1997, p. 259-266.

4. Cf. Fukuō jiden 福翁自伝, 1899. Traduction française : La vie du vieux Fukuzawa racontée par luimême, trad. et annot. Marie-Françoise Tellier, Paris, Albin Michel, 2007, p. 58.

5. Cf. Nishikawa Shunsaku, "Fukuzawa Yukichi», Perspectives: revue trimestrielle d'éducation comparée, Unesco, Bureau international d'éducation, vol. XXIII, nº 3-4, sept.-déc. 1993, p. 501-515. 6. «L'Appel à l'étude - livre premier », op. cit., p. 11-12. 
7. Keimō undō 啓蒙運動. Nous traduisons keimō, terme qui désigne les Lumières et l'instruction, par Aufklärung (éclairer, éclaircir) dans la mesure notamment où la plupart des intellectuels de l'époque Meiji préoccupés de la construction d'un État moderne s'intéressèrent surtout aux philosophies allemandes.

8. 通俗国権論; Fukuzawa Yukichi senshū 福沢諭吉撰集 (Euvres choisies de Fukuzawa Yukichi), Iwanami Shoten 岩波書店, vol. VII, (1980), 1989.

9. Anne Cheng, Histoire de la pensée chinoise, Éditions du Seuil, 2002.

10. Nihon seiji shisōshi kenkyū 日本政治思想史研究, traduit par Jacques Joly, Essai sur l'histoire des idées politiques au Japon, P.U.F, 1996. À propos du développement de la nouvelle tradition confucéenne dans la société japonaise, Frédéric Girard, Annick Horiuchi, Mieko Macé (dir.), Repenser l'ordre, repenser l'héritage. Paysage intellectuel du Japon (XVII ${ }^{e}$-XVIII ${ }^{e}$ siècles), Genève, École Pratique des Hautes Études Orientales, 2002.

11. Nihon seiji shisōshi kenkyū, traduction op. cit., p. 21.

12. Uno Shigeki, « La Modernisation politique au Japon et l'idée de subjectivité chez Maruyama Masao », Ebisu, n 32, Printemps-Eté 2004, p. 67-83.

13. Chō-kokkashugi no ronri to shinri 超国家主義の論理と心理, Sekai 世界, mai 1946. 